\title{
Complete and Partial Loss of Entanglement Due to a Phonon-Assisted Dephasing Process
}

\author{
K. Roszak, P. Machnikowski and L. JaCAK \\ Institute of Physics, Wrocław University of Technology \\ Wybrzeże Wyspiańskiego 27, 50-370 Wrocław, Poland
}

\begin{abstract}
We study the evolution of entanglement between two excitons in a double quantum dot system coupled to a super-Ohmic reservoir. As expected entanglement is more fragile than local coherence, but, surprisingly, for a set of pure states disentanglement can be complete in a finite time under conditions that lead to the usual partial pure-dephasing.
\end{abstract}

PACS numbers: 03.67.Lx, 03.65.Yz, 63.20.Kr

\section{Introduction}

Decoherence processes attract much attention as a part of the study of open quantum systems and for more application-oriented reasons (e.g., the implementation of quantum information technology). Quantum dots, for instance, are susceptible to a pure dephasing effect that has been experimentally observed [1] and theoretically explained by the phonon response to an ultra-fast change of the confined charge distribution $[2,3]$. In semiconductor quantum dot systems, the lattice response leads to a partial pure dephasing on the time scales of a few picoseconds [1], followed by an exponential decay of coherence on much longer time scales.

Sustaining coherence in multi-component systems is vital for maintaining entanglement, a key component of all quantum computing schemes $[4,5]$. An entangled state is a non-classical superposition state of two subsystems that, in principle, may be separated by an arbitrary distance, and thus its particular fragility under decoherence is expected. Indeed, even partial dephasing may lead to a complete disentanglement as has been shown for two classes of systems $[6,7]$.

\section{The system}

We study a system consisting of two excitons in one or two self-assembled semiconductor quantum dots (if we are dealing with one dot, the distance between 
the dots $d$ is equal to zero). A subsystem state $|0\rangle$ denotes an empty dot, while the state $|1\rangle$ means that an exciton is present. The charge carriers interact with acoustic phonons by deformation potential coupling [8]. The Hamiltonian of this system is

$$
\begin{gathered}
H=\epsilon_{1}(|1\rangle\langle 1| \otimes \mathcal{I})+\epsilon_{2}(\mathcal{I} \otimes|1\rangle\langle 1|)+\Delta \epsilon(|1\rangle\langle 1|\otimes| 1\rangle\langle 1|) \\
+(|1\rangle\langle 1| \otimes \mathcal{I}) \sum_{\boldsymbol{k}} f_{\boldsymbol{k}}^{(1)}\left(b_{\boldsymbol{k}}^{\dagger}+b_{-\boldsymbol{k}}\right)+(\mathcal{I} \otimes|1\rangle\langle 1|) \sum_{\boldsymbol{k}} f_{\boldsymbol{k}}^{(2)}\left(b_{\boldsymbol{k}}^{\dagger}+b_{-\boldsymbol{k}}\right)+\sum_{\boldsymbol{k}} \omega_{\boldsymbol{k}} b_{\boldsymbol{k}}^{\dagger} b_{\boldsymbol{k}} \cdot(1)
\end{gathered}
$$

The first two terms describe the single excitons, with their energies denoted by $\epsilon_{1,2}$ ( $\mathcal{I}$ is the unit operator), and the third accounts for their interaction ( $\Delta \epsilon$ is the biexciton energy shift). The next two terms in the Hamiltonian describe the exciton-phonon coupling, where $f_{\boldsymbol{k}}^{(1,2)}$ are system-reservoir coupling constants, and $b_{\boldsymbol{k}}, b_{\boldsymbol{k}}^{\dagger}$ are bosonic operators of the reservoir modes. The last term is the reservoir Hamiltonian; $\omega_{\boldsymbol{k}}$ is the energy (we put $\hbar=1$ ) of mode $\boldsymbol{k}$. The explicit tensor notation refers to the two subsystems but is suppressed for the reservoir components.

The time evolution of the system is calculated exactly using the Weyl operator method $[8,9]$ up to a local unitary rotation (conserving the amount of entanglement). The transformed density matrix may be written in the operator sum representation (see [10] for details)

$$
\rho(t)=\sum_{\mu} U K_{\mu} \rho(0) K_{\mu}^{\dagger} U^{\dagger},
$$

with the (non-local) unitary operator

$$
\begin{aligned}
U= & |0\rangle\langle 0|+\exp \left(\mathrm{i} \sum_{\boldsymbol{k}}\left|g_{\boldsymbol{k}}\right|^{2} \sin \omega_{\boldsymbol{k}} t\right)(|1\rangle\langle 1|+| 2\rangle\langle 2|) \\
& +\exp \left(4 \mathrm{i} \sum_{\boldsymbol{k}}\left|g_{\boldsymbol{k}}\right|^{2} \cos ^{2} \frac{k_{z} d}{2} \sin \omega_{\boldsymbol{k}} t-\mathrm{i} \Delta E t\right)|3\rangle\langle 3|,
\end{aligned}
$$

with $|0\rangle \equiv|0\rangle|0\rangle,|1\rangle \equiv|0\rangle|1\rangle$, etc., and the set of Kraus operators

$$
\begin{aligned}
& K_{0}=\operatorname{diag}[a(t), b(t), b(t), a(t)], \\
& K_{1}=\operatorname{diag}\left[\left(a^{2}(t)-1\right) \sqrt{a^{2}(t)+1}, 0,0,0\right], \\
& K_{2}=\sqrt{1-a^{2}(t)} \operatorname{diag}\left[-a^{2}(t), 0,0,1\right], \\
& K_{3}=\operatorname{diag}\left[0,0,\left(b^{2}(t)-1\right) \sqrt{b^{2}(t)+1}, 0\right], \\
& K_{4}=\sqrt{1-b^{2}(t)} \operatorname{diag}\left[0,1,-b^{2}(t), 0\right],
\end{aligned}
$$

where 


$$
\begin{aligned}
& a(t)=\exp \left(\sum_{\boldsymbol{k}}\left|g_{\boldsymbol{k}}\right|^{2} \cos ^{2} \frac{k_{z} d}{2}\left(\cos \omega_{\boldsymbol{k}} t-1\right)\left(2 n_{\boldsymbol{k}}+1\right)\right), \\
& b(t)=\exp \left(\sum_{\boldsymbol{k}}\left|g_{\boldsymbol{k}}\right|^{2} \sin ^{2} \frac{k_{z} d}{2}\left(\cos \omega_{\boldsymbol{k}} t-1\right)\left(2 n_{\boldsymbol{k}}+1\right)\right),
\end{aligned}
$$

and $g_{\boldsymbol{k}}=f_{\boldsymbol{k}} / \omega_{\boldsymbol{k}}$. The set of operators $K_{\mu}$ corresponds to a kind of two-qubit phase damping channel. This channel is generated by the same physical pure dephasing process that would lead to the usual phase-damping channel for a single qubit [11]. The operators $K_{1,2}$ may be interpreted as an effect of a "charge detector" which is sensitive only to the total number of excitons in the system. On the other hand, $K_{3,4}$ reflect the action of a "discriminator" which detects in which of the two dots the exciton is present. Since the two subsystems are identical they may be distinguished by the reservoir only because of their different position. Therefore, the latter contribution to dephasing is inefficient for $d=0$. For a system of two excitons confined in a single dot $(d=0)$ the present result reduces to that found in Ref. [12].

\section{Disentanglement}

To calculate the evolution of entanglement one needs an entanglement measure that can be calculated from the state of the system. For pure states the von Neumann entropy of one subsystem [13] is the unique entanglement measure but for mixed states there is a choice of measures [14-16]. One of them is the entanglement of formation (EOF), defined as the ensemble average of the von Neumann entropy minimized over all ensemble preparations of the state [16]. An explicit formula for calculating it exists only for a pair of two level systems [17, 18],

$$
E[\rho(t)]=-x_{+} \log _{2} x_{+}-x_{-} \log _{2} x_{-},
$$

where

$$
x_{ \pm}=\frac{1 \pm \sqrt{1-C^{2}[\tilde{\rho}(t)]}}{2}
$$

and $C[\tilde{\rho}(t)]$ is the concurrence, given by

$$
C[\tilde{\rho}(t)]=\max \left(0, \lambda_{0}-\lambda_{1}-\lambda_{2}-\lambda_{3}\right),
$$

where $\lambda_{i}$ are the square roots of eigenvalues of the matrix $\tilde{\rho}(t)\left(\sigma_{y} \otimes \sigma_{y}\right) \tilde{\rho}^{*}(t)\left(\sigma_{y} \otimes \sigma_{y}\right)$ in decreasing order ( $\sigma_{y}$ are Pauli matrices).

The calculations were performed for two initial, maximally entangled pure states

$$
\begin{aligned}
\left|\psi_{0}^{(1)}\right\rangle & =\frac{|01\rangle-|10\rangle}{\sqrt{2}} \\
\left|\psi_{0}^{(2)}\right\rangle & =\frac{|00\rangle+|01\rangle+|10\rangle-|11\rangle}{2}
\end{aligned}
$$

the first has only one coherence between single electron states, which is indistinguishable by phonons at $d=0$, while the second has all coherences present. 
The evolution of entanglement for these two states is shown for different temperatures, at a fixed distance between the $\operatorname{dots}(d=6 \mathrm{~nm})$ and no biexcitonic shift in Figs. 1a and d. As expected, in both cases building up of correlations with the phonon reservoir destroys the entanglement between the subsystems. For the initial state $(5 \mathrm{a})$ the disentanglement resembles the dephasing process and is only partial. For the other initial state, if the temperature is high enough, entanglement may vanish completely at a finite time. The dashed line in Fig. 1d corresponds to a non-zero biexcitonic shift $\left(\Delta E=6 \mathrm{ps}^{-1}\right)$ at $T=40 \mathrm{~K}$. Remarkably, extended periods of time when entanglement remains zero may be noted in this case.
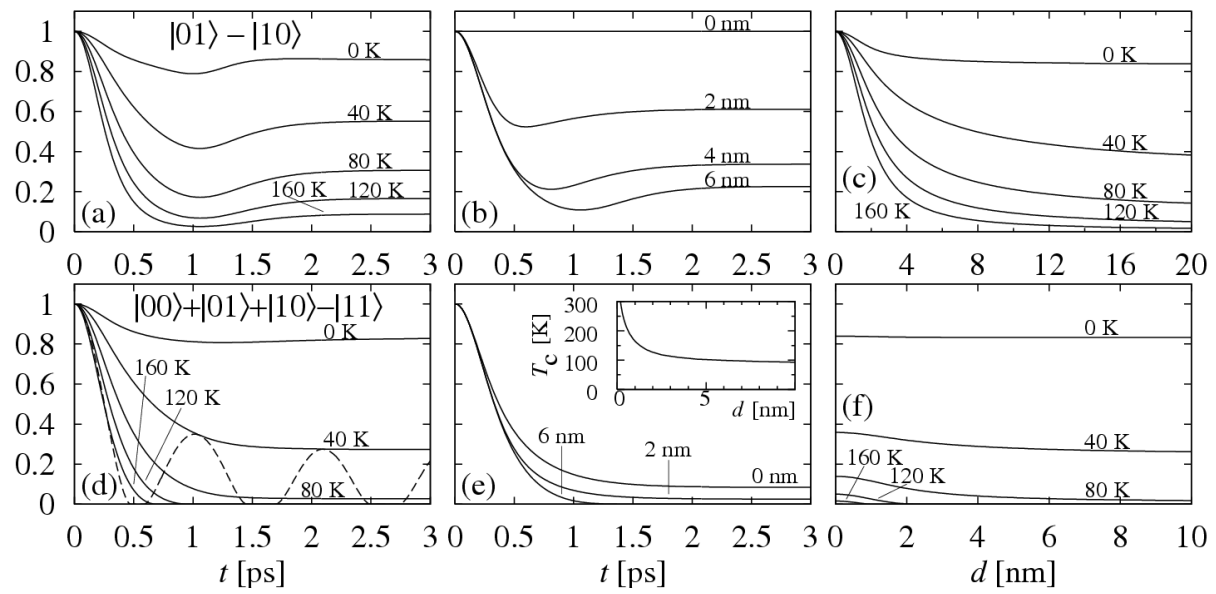

Fig. 1. The evolution of entanglement of two selected initial states for various temperatures and separations between subsystems (left panels: $d=6 \mathrm{~nm}$; middle panels: $T=100 \mathrm{~K})$ and the asymptotic, long time value of entanglement as a function of separation for a range of temperatures (right panels). The inset in (e) shows the temperature at which complete disentanglement appears as a function of separation.

Figures $1 b$ and e show the evolution of entanglement for different distances between the dots at a fixed temperature of $100 \mathrm{~K}$. Again, the destruction of entanglement in the evolution of the initial state $(5 \mathrm{a})$ is always only partial but the appearance of complete disentanglement for the initial state (5b) is dependent on the separation of the subsystems (large separations promote disentanglement). The inset in Fig. 1e shows the lowest temperature at which the decay becomes complete for a given temperature. The effect of separation is more clearly seen in Figs. 1c and f which show the dependence of the asymptotic value of entanglement on the distance $d$ for a set of temperatures. The state $(5 \mathrm{a})$ shows robust entanglement when both excitons are located in one quantum dot (QD) $(d=0)$, because the components of the superposition cannot be distinguished by the phonon reservoir, so no dephasing effect is possible. For non-zero distances the entanglement is fragile. The initial state $(5 \mathrm{~b})$ is fragile independently from the distance between the 
dots, but for $d=0$ complete disentanglement is not possible (this is because some components of the superposition are not distinguishable by the phonon reservoir). These different behaviors, apparent for both initial states, reflect the crossover between the common-reservoir regime and the regime of independent reservoirs [19].

The appearance of complete disentanglement for some initial states under sufficiently strong partial pure dephasing may be understood with the help of Eq. (4). If the completely dephased state (with a diagonal density matrix) has $\lambda_{0}-\lambda_{1}-\lambda_{2}-\lambda_{3}<0$ then, by continuity, it will be surrounded by states with vanishing concurrence, thus separable. In this case entanglement vanishes for sufficiently strongly dephased states, before the complete dephasing is reached. For a diagonal density matrix one finds $\lambda_{0}-\lambda_{1}-\lambda_{2}-\lambda_{3}=-2 \min \left(\rho_{00} \rho_{33}, \rho_{11} \rho_{22}\right)$, so that the above condition may only be satisfied if all four diagonal elements are nonzero. For instance, the totally mixed state (with a density matrix proportional to unity) is surrounded by a ball of separable states [20]. Out of the initial states considered here, the first one (Eq. (5a)) satisfies the above condition (it decays towards the totally mixed state) but the second one (Eq. (5b)) does not. In general, the structure of entangled and separable states around the final state may be quite complicated.

\section{Conclusion}

We have studied the evolution of entanglement between two excitons confined in one or two QDs coupled to a phonon reservoir. The correlations between the system and the reservoir that lead to pure dephasing destroy the entanglement, and the effect depends on the initial state, temperature, and the distance between the subsystems. For a class of initial states, the disentanglement may even be complete at a finite time, even though the dephasing is always only partial. It should be stressed that the appearance of complete disentanglement at increased temperatures is related to a stronger dephasing, and, in principle, a state may become separable already at $0 \mathrm{~K}$ if the coupling would be sufficiently strong. The dependence on the distance between the quantum dots, however, reflects a more fundamental crossover from the common-reservoir regime to that of independent reservoirs.

\section{References}

[1] P. Borri, W. Langbein, S. Schneider, U. Woggon, R.L. Sellin, D. Ouyang, D. Bimberg, Phys. Rev. Lett. 87, 157401 (2001).

[2] A. Vagov, V.M. Axt, T. Kuhn, Phys. Rev. B 67, 115338 (2003).

[3] L. Jacak, J. Krasnyj, W. Jacak, R. Gonczarek, P. Machnikowski, Phys. Rev. B 72, 245309 (2005).

[4] E. Biolatti, R.C. Iotti, P. Zanardi, F. Rossi, Phys. Rev. Lett. 85, 5647 (2000).

[5] T. Calarco, A. Datta, P. Fedichev, E. Pazy, P. Zoller, Phys. Rev. A 68, 12310 (2003). 
[6] T. Yu, J.H. Eberly, Phys. Rev. Lett. 93, 140404 (2004).

[7] P.J. Dodd, J.J. Halliwell, Phys. Rev. A 69, 052105 (2004).

[8] G.D. Mahan, Many-Particle Physics, Kluwer, New York 2000.

[9] K. Roszak, P. Machnikowski, Phys. Lett. A 351, 251 (2006).

[10] K. Roszak, P. Machnikowski, Phys. Rev. A 73, 022313 (2006).

[11] J. Preskill, Quantum Information and Computation, lecture notes avaliable at http://www.theory.caltech.edu/preskill/ph229 (unpublished).

[12] V.M. Axt, P. Machnikowski, T. Kuhn, Phys. Rev. B 71, 155305 (2005).

[13] C.H. Bennett, H.J. Bernstein, S. Popescu, B. Schumacher, Phys. Rev. A 53, 2046 (1996).

[14] C.H. Bennett, G. Brassard, S. Popescu, B. Schumacher, J.A. Smolin, W.K. Wootters, Phys. Rev. Lett. 76, 722 (1996).

[15] C.H. Bennett, D.P. DiVincenzo, J.A. Smolin, W.K. Wootters, Phys. Rev. A 54, 3824 (1996).

[16] M. Horodecki, Quant. Inf. Comp. 1, 3 (2001).

[17] S. Hill, W.K. Wootters, Phys. Rev. Lett. 78, 5022 (1997).

[18] W.K. Wootters, Phys. Rev. Lett 80, 2245 (1998).

[19] T. Yu, J.H. Eberly, Phys. Rev. B 66, 193306 (2002).

[20] K. Życzkowski, P. Horodecki, A. Sanpera, M. Lewenstein, Phys. Rev. A 58, 883 (1998). 